La Revue

des Droits

de l'Homme

\section{La Revue des droits de l'homme}

Revue du Centre de recherches et d'études sur les droits fondamentaux

Actualités Droits-Libertés | 2019

\title{
Face au bulldozer « ELAN », le Conseil constitutionnel reste de béton
}

À propos de la décision n ${ }^{\circ} 2018-772$ DC du 15 novembre 2018, Loi portant évolution du logement, de l'aménagement et du numérique

\section{Patricia Rrapi}

\section{OpenEdition}

\section{Journals}

Édition électronique

URL : http://journals.openedition.org/revdh/5702

DOI : $10.4000 /$ revdh.5702

ISSN : 2264-119X

\section{Éditeur}

Centre de recherches et d'études sur les droits fondamentaux

\section{Référence électronique}

Patricia Rrapi, «Face au bulldozer «ELAN », le Conseil constitutionnel reste de béton », La Revue des droits de l'homme [En ligne], Actualités Droits-Libertés, mis en ligne le 08 janvier 2019, consulté le 19 avril 2019. URL : http://journals.openedition.org/revdh/5702 ; DOI : 10.4000/revdh.5702

Ce document a été généré automatiquement le 19 avril 2019

Tous droits réservés 


\section{Face au bulldozer « ELAN », le Conseil constitutionnel reste de béton}

À propos de la décision n 2018-772 DC du 15 novembre 2018, Loi portant évolution du logement, de l'aménagement et du numérique

\section{Patricia Rrapi}

1 Contestée, discutée, fortement amendée, la loi ELAN, finalement adoptée par le Parlement, a été également validée, dans ses principes, par le Conseil constitutionnel. Or, au-delà de l'assouplissement des contraintes établies au cours des quarante dernières années en matière de construction, les principes de la loi ELAN engagent une véritable philosophie de l'espace. On pourrait s'étonner d'ailleurs de la franchise avec laquelle, malgré le contexte actuel, celui d'un autre "élan », plutôt en faveur de la protection de l'environnement, le titre premier de la loi affiche son objectif : «construire plus, mieux et moins cher ». Si, à première vue, nous avons du mal à voir dans cette phrase un simple slogan des promoteurs de l'immobilier, c'est parce que le discours justificatif de la loi, que le Conseil constitutionnel a repris dans sa décision, est mené sur le terrain du "pragmatique ». Pour quelle raison seulement $20 \%$, au lieu de $100 \%$, des bâtiments nouveaux doivent-ils être accessibles aux personnes à mobilité réduite? C'est évident : non seulement les personnes à mobilité réduite sont une minorité sur le territoire, mais ces contraintes empêchent la construction de logements pour la majorité de la population ${ }^{1}$. Opposer majorité et minorité est un argument classique. Pour quelle raison doit-on assouplir la " loi littoral " ? C'est évident là encore : la construction des logements à côté de la mer permet d'améliorer l'offre de logement. Opposer protection de l'environnement et bien-être social est un argument, là aussi, classique.

2 C'est la raison pour laquelle les députés auteurs de la saisine ont cru pouvoir défendre, en saisissant le Conseil constitutionnel en dernier ressort, d'autres arguments. Arguer, par exemple, que le mètre carré des logements ne doit pas être conçu en fonction des intérêts économiques des promoteurs de l'immobilier mais être pensé à la lumière du bien-être et de l'espace de vie intérieur de tous les citoyens, à mobilité réduite ou non; que l'espace 
que nous construisons doit être pluridimensionnel et non pas stéréotypé; qu'il existe beaucoup d'endroits pour construire des logements ailleurs que sur le littoral. Ce raisonnement aussi est simple, tellement simple que le Conseil constitutionnel, pour valider la loi, devait l'éviter en reprenant les oppositions (minorité/majorité; environnement/social) offertes par le gouvernement. Mais pour le Conseil, il fallait également intégrer ces oppositions dans une interprétation supplémentaire des textes de références. Ainsi, afin de préserver une certaine « cohérence » de sa décision, le Conseil ne pouvait que s'enliser dans un raisonnement sélectif, périphérique et, finalement, expéditif.

\section{Le raisonnement sélectif}

3 Le Conseil constitutionnel n'a pas vocation à l'humour ; ses décisions prêtent cependant souvent à sourire. En l'espèce, si le Conseil constitutionnel rattache, pour la première fois ${ }^{2}$, la «mise en œuvre d'une politique de solidarité nationale en faveur des personnes handicapées » aux alinéas 11 et 12 du Préambule de la Constitution de 1946, ce n'est que pour mieux valider la loi qui fait passer l'exigence d'accessibilité pour les bâtiments nouveaux de $100 \%$ à $20 \%$. En réalité, les auteurs de la saisine défendaient l'idée selon laquelle de ces deux alinéas découle le «principe d'accessibilité au logement des personnes à mobilité réduite", qui limiterait ainsi la possibilité pour le législateur de réduire le pourcentage de bâtiments accessibles - si ce n'est de manière absolue, au moins pas de façon aussi drastique. En «constitutionnalisant» la «politique de solidarité nationale en faveur des personnes handicapées", le Conseil fait du principe d'accessibilité une simple modalité de mise en œuvre de cette politique, laissant au gouvernement la possibilité de supprimer, adapter, etc., "à tout moment», les dispositions législatives favorables aux personnes handicapées :

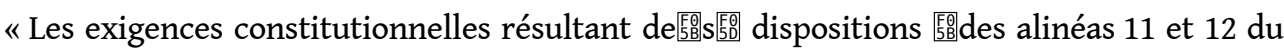
Préambule de la Constitution de 1946㺝 impliquent la mise en œuvre d'une politique de solidarité nationale en faveur des personnes handicapées. Il est cependant possible au législateur, pour satisfaire à ces exigences, de choisir les modalités concrètes qui lui paraissent appropriées. En particulier, il lui est à tout moment loisible, statuant dans le domaine qui lui est réservé par l'article 34 de la Constitution, de modifier des textes antérieurs ou d'abroger ceux-ci en leur substituant, le cas échéant, d'autres dispositions. Il ne lui est pas moins loisible d'adopter, pour la réalisation ou la conciliation d'objectifs de nature constitutionnelle, des modalités nouvelles dont il lui appartient d'apprécier l'opportunité et qui peuvent comporter la modification ou la suppression de dispositions qu'il estime excessives ou inutiles. Cependant, l'exercice de ce pouvoir ne saurait aboutir à priver de garanties légales des exigences de caractère constitutionnel. » (Cons. 36).

5 En effet, un tel considérant serait inutile s'il visait, pour le futur, les mesures législatives favorables à la "politique de solidarité nationale en faveur des personnes handicapées ». Comme en l'espèce, cette approche permet surtout de valider les mesures législatives futures défavorables aux personnes handicapées. Quant à la limite qui consiste à ne pas priver de " garanties légales » les exigences constitutionnelles, elle est aussi entendue de manière très souple, à partir du moment où le passage de 100 à $20 \%$ de bâtiments accessibles est validé et que le principe d'accessibilité au logement n'est pas, en tant que tel, une « garantie légale ». 
Il semble toutefois que cette stratégie de classement-déclassement ne pouvait à elle seule justifier la décision de conformité compte tenu du caractère vertigineux de la chute du pourcentage de bâtiments accessibles. Non seulement parce que les auteurs de la saisine invoquaient également la violation du principe d'égalité, mais aussi parce que la jurisprudence sur les "garanties légales " pouvait s'avérer tout de même, en l'espèce, contraignante, aussi bien pour le législateur que pour le Conseil constitutionnel luimême. C'est alors une autre stratégie qui est déployée. Afin de valider la loi, le Conseil constitutionnel mobilise, à la suite du gouvernement, la notion de logement "évolutif ", permettant la transformation des logements, après "simples travaux ", en logements accessibles. Cette garantie légale que constituent les « logements évolutifs » se substitue à la garantie légale du pourcentage. Or, au-delà du flou que comporte cette garantie légale de substitution, le caractère discriminatoire d'une telle disposition - le coût des travaux, la position défavorable des personnes handicapées sur le marché de la location - n'a pas retenu l'attention du Conseil car, si le principe d'égalité est invoqué par les auteurs de la saisine, le Conseil ne fait que l'écarter au détour d'une unique phrase (cons. 41). Il préfère ainsi concentrer son contrôle sur la «politique de la solidarité nationale en faveur de personnes handicapées » et ne donner aucun argument quant à la conformité de la disposition au principe d'égalité. C'est dans le commentaire officiel que le Conseil expose, en revanche, la raison-perle de la conformité de la loi au principe d'égalité: la loi, précise-t-on, impose de « manière uniforme des règles pour la construction des bâtiments d'habitation collectifs $»^{3}$. Il est de ces phrases qui s'efforcent si bien de ne rien dire qu'elles finissent par en exprimer beaucoup, moins du sujet initial que de l'idée sousjacente que se fait le locuteur, en l'espèce, tant de la politique du handicap, tout juste constitutionnalisée, que du principe d'égalité ou encore des lecteurs des décisions et commentaires officiels du Conseil constitutionnel eux-mêmes... En réalité, la raison principale pour laquelle le Conseil préfère les alinéas 11 et 12 du Préambule au principe d'égalité est donnée dans le commentaire officiel: «si la valeur constitutionnelle de ces deux alinéas a bien été reconnue par le Conseil constitutionnel, celui-ci a jusqu'à présent circonscrit la portée des obligations en résultant pour le législateur $»^{4}$. En résumé, invoquer le Préambule de 46 est devenu presque un piège pour les requérants, comme l'est encore davantage le grief tiré de la violation de la Charte de l'environnement.

\section{Le raisonnement périphérique}

7 Belle tentative des auteurs de la saisine de faire interpréter la Charte de l'environnement à la lumière de l'Accord de Paris sur le climat. C'est oublier cependant la manière dont les décisions récentes ont consolidé le silence dans lequel la Charte a été murée depuis son entrée en vigueur ${ }^{5}$.

Cette fois, ce sont les auteurs de la saisine qui proposaient d'interpréter la « loi littoral » comme une base légale des exigences constitutionnelles découlant de l'article $1^{\text {er }}$ de la Charte de l'environnement qui consacre le droit de vivre dans un environnement sain. Il s'agissait donc de consacrer, via la jurisprudence sur les "garanties légales », un verrou principe de non-régression - sur une législation protectrice de l'environnement, qui plus est, en l'espèce, la plus emblématique.

9 La question posée au Conseil constitutionnel était en effet épineuse, non seulement parce que l'assouplissement de la « loi littoral » a fait l'objet de vifs débats lors de son adoption, mais aussi parce qu'une régression en matière de protection de l'environnement et plus 
particulièrement de protection d'une zone exposée comme le littoral, pose, au-delà même du bon sens, une sérieuse question de conformité à la Charte. Le Conseil constitutionnel refuse cependant de voir dans la «loi littoral» une garantie légale des exigences constitutionnelles découlant de l'article 1 de la Charte, comme il a pu le faire ailleurs ${ }^{6}$. Mais cette décision ne fait en réalité que confirmer la marginalisation constante de la Charte dans le contentieux constitutionnel. Il s'agit là d'une volonté claire de ne pas lier une législation protectrice de l'environnement à la Charte, déconnectant le contenu de celle-ci de toute tentative, parfois même antérieure, comme en l'espèce, à la Charte ellemême, de protéger l'environnement. La solution pour le Conseil consiste alors à ne pas répondre à la question de savoir si une régression législative en matière de protection de l'environnement porte atteinte à l'article 1 de la Charte. Le Conseil se contente de rester à la périphérie du problème en " analysant » les "limites " posées par le législateur à une telle régression. Le commentaire officiel, quant à lui, fait le choix délibéré de ne pas évoquer du tout la décision du Conseil constitutionnel sur ce point. Il ne faut cependant pas voir dans cette posture du Conseil constitutionnel une réticence à l'égard des politiques proactives en matière de protection de l'environnement, mais plutôt une tendance à vouloir isoler la Charte de l'environnement qui ne pourrait ainsi le lier pour le futur. En d'autres termes, le Conseil constitutionnel aurait peut-être voulu censurer la loi, mais cela l'aurait obligé à interpréter la Charte.

\section{Le raisonnement expéditif}

Le Conseil constitutionnel tend à se montrer souvent, voire très souvent, expéditif dans ses raisonnements mais, dans le cadre de l'application de la Charte de l'environnement, cette tendance prend des dimensions grotesques. S'agissant du grief tiré de la violation de l'obligation de préserver l'environnement (art.2) et du principe de précaution (art.5), le Conseil répète machinalement à quatre reprises et pour des dispositions diverses le considérant suivant : " ces dispositions, qui ne méconnaissent ni les articles 2 et 5 de la Charte de l'environnement ni aucune autre exigence constitutionnelle, sont conformes à la Constitution » (nous soulignons). Il faut donc croire le Conseil constitutionnel sur parole.

11 Face à ce silence, couvert également par le commentaire officiel, deux hypothèses s'imposent. Écartons cependant la première hypothèse, selon laquelle il existerait un réel problème de constitutionnalité que le Conseil souhaiterait masquer par une réponse expéditive, même si sur ce point la saisine des députés est la plus argumentée et la mieux fournie en informations. En effet, le Conseil en a tellement peu dit, à ce jour, sur le principe de précaution (art.5) et sur l'obligation de préserver l'environnement (art. 2) qu'il lui est possible de valider n'importe quelle mesure. La seconde hypothèse mérite davantage l'attention. À partir du moment où les dispositions législatives sont conformes aux articles 2 et 5 de la Charte, le Conseil aurait pu, ne serait-ce que pour conforter la constitutionnalité des dispositions examinées comme il le fait pour l'article $1^{\text {er }}$ de la Charte, fournir quelques arguments. Mais, s'agissant du principe de précaution, le Conseil semble vouloir neutraliser toutes les interprétations, fussent-elles a contrario. En ne donnant aucun argument pour conforter sa décision actuelle, le Conseil évite que des " arguments incidents " puissent le lier d'une façon ou d'une autre dans une décision future. Procède de cette même logique la censure de plusieurs articles de la loi pour violation de la procédure législative - «cavaliers législatifs ». Moins on en dit, mieux on 
se protège de la potentialité contraignante de la Charte de l'environnement en général et du principe de précaution en particulier. C'est, là, une véritable précaution.

CC, Décision n 2018-772 du 15 novembre 2018 - Loi portant évolution du logement, de l'aménagement et du numérique.

Les Lettres «Actualités Droits-Libertés»(ADL) du CREDOF (pour s'y abonner) sont accessibles sur le site de la Revue des Droits de l'Homme (RevDH) - Contact

\section{NOTES}

1. Voir sur ce point le commentaire officiel.

2. Commentaire officiel de la décision p. 7

3. Commentaire officiel p. 8.

4. Commentaire officiel p. 5.

5. Voir décision $n^{\circ}$ 2018-768 DC (cons. 27) ; décision $n^{\circ}$ 2017-749 DC (cons. 15).

6. Voir par exemple à propos de la Loi informatique et liberté (1978) devenue une garantie légale de l'exigence constitutionnelle du droit au respect de la vie privée (décision n ${ }^{\circ}$ 92-316 DC (cons. 14) ou encore décision 2013-648 DC (cons.10).

\section{RÉSUMÉS}

Contestée, discutée, fortement amendée, la loi ELAN, finalement adoptée par le Parlement, a été également validée, dans ses principes, par le Conseil constitutionnel. Or, au-delà de l'assouplissement des contraintes établies au cours des quarante dernières années en matière de construction, les principes de la loi ELAN engagent une véritable philosophie de l'espace

\section{AUTEUR}

\section{PATRICIA RRAPI}

Maitre de conférences à l'Université Paris Nanterre 